\title{
Viewpoints: an interactive forum for debates and discussions
}

\author{
Neuroscientists dedicate themselves to understanding the brain. But what happens when they disagree on \\ experimental outcomes, data interpretation or methodology? Nature Neuroscience debuts a format that invites \\ researchers to debate critical issues in neuroscience.
}

W hen issues arise that divide researchers in a field, they often are addressed as passing acknowledgments in the discussion section of research papers and will perhaps arise as points of spirited discussion at conferences and workshops. Live debates are stimulating and exciting, but the unfortunate downside is that the discussion is limited to those in attendance (except in the rare instance when the event is recorded; for example, https://www.cogneurosociety.org/watch-the-great-debate-connectomics/). Further, a detailed record of the finer discussion points is often missing. And as people go back to the lab to pursue their primary research interests, potential avenues for collaborations that may have emerged from these interactions may not be explored, leaving these issues unresolved and impeding scientific progress.

In an effort to bring the spirit of a live discussion to a written forum, Nature Neuroscience is launching a Viewpoints format. In these pieces, a moderator provides a framework to discuss a pressing issue in the field with a series of questions presented to a panel of experts with diverging opinions on the topic. The Viewpoints moderator is carefully selected by the editorial team; this person must work in the relevant field and be familiar with the issues at hand, but must be sufficiently neutral as to be able to encourage a robust debate without favoring one of the views. The editorial team works with the moderator to develop the questions that will be presented to the panelists, highlighting the points of contention while leading the panelists towards the possibility of integrating different opinions. The panelists are active researchers who represent diverging opinions, distinct institutions and varying research interests. After reviewing the panelists' responses, the editor and moderator can propose additional questions and provide the panelists with the opportunity to interact by presenting follow-up points and questions to each other. The moderator wraps up the debate with a closing statement on themes that have emerged from the interactive discussion before working with the editor to select the most salient discussion points to present in the final published dialogue. For the particularly keen readers, we will also publish the unabridged discourse as a supplement alongside the panelists' brief essays that summarize each viewpoint represented in the discussion, so that readers can learn more about the research supporting the conflicting perspectives.

Our first Viewpoints, on page 1545, is led by Mauricio Delgado, who brings together human and animal researchers in economic valuation (Lesley Fellows and Scott Huettel), affective regulation (Daniela Schiller and Gregory Quirk) and social cognition (Jennifer Beer and Michael Platt) to discuss the functions attributed to the ventromedial prefrontal cortex. We hope that you enjoy reading the dialogue and considering the evidence that supports each viewpoint and the ways in which potential integration of these viewpoints might be achieved.

We hope that this new format will not only highlight contentious issues across several disciplines of neuroscience, but also inspire new research collaborations that may move the field closer to resolving them. And we encourage you to take part in this endeavor. Please send your ideas for other topics that you would like to see covered in our Viewpoints to us at neurosci@us.nature.com. Science is a dialogue, and increased exchange leads to greater understanding. Join us in the discussion. 